\title{
A SPLITTING THEOREM FOR 4-DIMENSIONAL MANIFOLDS OF NONNEGATIVE CURVATURE
}

\author{
GERARD WALSCHAP \\ (Communicated by David G. Ebin)
}

\begin{abstract}
A structure theorem for four-dimensional open manifolds of nonnegative curvature is stated. More generally, it is shown that any manifold with soul $S$ of codimension 2 admits a Riemannian submersion onto $S$, which splits as a metric product whenever $S$ has flat normal bundle.
\end{abstract}

Let $M^{n}$ denote a complete noncompact Riemannian manifold with nonnegative sectional curvature $K$. The metric structure of $M$ is well understood if $n \leq 4$, cf. $[\mathbf{C G}]$, except when the soul is 2-dimensional. This last case also relates to what could be called the "open" Hopf conjecture: does $S^{2} \times R^{2}$ admit a metric of nonnegative curvature that is strictly positive at some point? In earlier work, it was shown that if $M^{n}$ has a soul $S$ of codimension 2, then either the normal bundle $\nu(S)$ of $S$ is flat, or there is a Riemannian submersion $\pi: M \rightarrow S, \mathrm{cf}$. [W]. In this paper, we prove that if a codimension 2 , simply connected soul has flat normal bundle, then $M$ splits isometrically as $S \times P_{2}$, where $P_{2}$ denotes $R^{2}$ together with some metric of nonnegative curvature. Consequently, any $M$ of dimension $\leq 4$ admits a Riemannian submersion onto its soul, and if $K$ is strictly positive at some point, then the manifold is diffeomorphic to Euclidean space. The author is indebted to D. Gromoll for his many comments and suggestions.

The reader is referred to [CG] for the basic properties of nonnegative curvature. Suppose $M^{n}$ has a simply connected soul of codimension 2 with flat normal bundle. Reindex the family $\left\{C_{t}\right\}$ of totally convex sets in the soul construction if necessary, so that $\operatorname{dim} C_{0}<n$. Thus, either $C_{0}$ has dimension $n-1$, or $C_{0}=S$. Let $\gamma$ denote a minimal unit-speed connection from $S$ to some $\partial C_{t}, t>0$. Then $\gamma$ has length $t$, and the extension of $\gamma$ to any interval $\left[0, t^{\prime}\right]$ is a minimal connection to $\partial C_{t^{\prime}}$. In particular, $\gamma$ is a ray. Since $\nu(S)$ is flat and $S$ is simply connected, there exists a global parallel section $X$ of $\nu$ with $X_{\gamma(0)}=\dot{\gamma}(0)$. By [CG, Theorem 1.10], the map $f_{X}: S \times[0, \infty) \rightarrow M$, given by $f_{X}(p, s):=\exp s X_{p}$, is a 1-1 totally geodesic isometric immersion. Such a hypersurface will be called a branch of $M$. Since $S$ is at maximal distance from $\partial C_{t}$, a straightforward application of [CG, Lemma 4.1] shows that we can find 2 or 3 branches, such that any 2 adjacent ones meet at $S$ at an angle $\leq \pi$. It is easily seen that these then partition $C_{t}$ into (not necessarily totally) convex sets.

We are now ready to construct a foliation of $M$ by submanifolds isometric to $S$. One way to achieve this is to consider the soul of each convex component $D_{t}$ of $C_{t}$, and repeat the above construction inductively. Here we shall adopt a

Received by the editors September 17, 1987.

1980 Mathematics Subject Classification (1985 Revision). Primary 53C20. 
different approach, one that uses results rooted in the work of Sharafutdinov and independently Croke and Schroeder, cf. [Y].

Proposition 1 (Proposition 3.2, [Y $\mathbf{Y}]$ ). Let $S$ be a soul of $M$. Then $S$ has minimal volume in its homology class.

As pointed out in $[\mathbf{Y}]$, this result implies that if $X$ is any parallel section of $\nu(S)$, then $\varphi_{X}: S \times R \rightarrow M, \varphi_{X}(p, s):=\exp s X_{p}$, is a totally geodesic isometric immersion, since by the Rauch comparison theorem, $\varphi_{X}(\cdot, s)$ is distance non-increasing for small $s$.

LEMMA 2. There exists a smooth foliation of $M$ by totally geodesic submanifolds isometric to $S$.

ProOF. For each parallel section $X$ of unit length in $\nu$, let $\varphi_{X}: S \times[0, \infty) \rightarrow$ $M$ be as above. If $q \in M$ is realized as $\varphi_{X}\left(p, t_{0}\right)$, define the leaf through $q$ to be $\varphi_{X}\left(S \times t_{0}\right)$. It is a totally geodesic manifold isometric to $S$ by the previous observation. To see that the foliation is well defined, suppose $\operatorname{im} \varphi_{X}$ and $\operatorname{im} \varphi_{Y}$ intersect at $q$ outside $S$. Since they are both hypersurfaces, the intersection of their tangent spaces has dimension $\geq n-2$. Thus, if this intersection does not coincide with both copies of $S$, one obtains a geodesic lying in both hypersurfaces, connecting $S$ to $q$. This implies that $X=Y$, and the foliation is well defined. In particular, if $D_{t}$ is as above, and if $q \in D_{t}$, then the leaf through $q$ is entirely contained in $D_{t}$. Notice, however, that there can be at most one totally geodesic manifold $S^{\prime}$ isometric to $S$ with $D_{t} \supset S^{\prime}$, and $q \in S^{\prime}$. This is clear if $q$ belongs to some branch $\operatorname{im} f_{X}$. Otherwise, replacing $t$ if necessary by sufficiently large $t^{\prime}>t$, we may assume that $d\left(q, \partial D_{t}\right)=d\left(q\right.$, im $\left.f_{X}\right)$. Since $S^{\prime}$ is totally geodesic, it lies at constant distance from the boundary of $D_{t}$, by convexity of the distance function. If $c:[0, a] \rightarrow D_{t}$ is a minimal geodesic from $q$ to im $f_{X}$, with $c(a)=f_{X}\left(p, t_{0}\right)$, then the parallel translate of $S_{q}^{\prime}$ along $c$ is contained in the tangent space of the branch $S \times R$, where we have omitted $f_{X}$ for brevity. In fact, this parallel translate must coincide with the tangent space of $S \times t_{0}$, for otherwise some geodesic in $S^{\prime}$ would go to infinity. Let $Y$ be the parallel section of $\nu\left(S \times t_{0}\right)$ with $Y_{c(a)}=-\dot{c}(a)$, and let $\varphi_{Y}: S \times t_{0} \rightarrow M, \varphi_{Y}\left(r, t_{0}\right):=\exp a Y_{\left(r, t_{0}\right)}$. Then $S^{\prime}=\varphi_{Y}\left(S \times t_{0}\right)$, thereby proving uniqueness. It is now easy to check smoothness of the foliation: given $p \in M$, let $L$ be the leaf containing $p . L$ has flat normal bundle since by construction it admits one parallel section, and $\operatorname{codim} L=2$. If $p$ lies in some branch, choose $\varepsilon$ smaller than the injectivity radius of $\nu(L)$. Otherwise, take $\varepsilon<\min \left\{d\left(p, \partial D_{t}\right)\right.$, inj. radius of $\nu(L)\}$, where $D_{t}$ is the convex set containing $p$. Exponentiating the global parallel sections of $\nu(L)$ at distances $<\varepsilon$ yields totally geodesic manifolds isometric to $L$ (here again we use the fact that $L$ has minimal volume in its homology class), and each of them lies in some convex $D_{t}$. By uniqueness, these manifolds coincide with the leaves, thus proving smoothness of the foliation.

THEOREM 3. Let $M^{n}$ have a simply connected soul $S$ of codimension 2, with flat normal bundle. Then $M=S \times P_{2}$ isometrically, where $P_{2}$ is $R^{2}$ together with some metric of nonnegative curvature.

ProOF. It was shown in the proof of 1.2 that the leaves of the foliation $F$ are equidistant. It follows easily that $M / F$ is a smooth manifold and admits a 
Riemannian metric for which the projection $\pi: M \rightarrow M / F$ becomes a Riemannian submersion, cf. also [H]. The fibers of $\pi$ are totally geodesic and simply connected, and the O'Neill tensor $A$ vanishes identically (if $X$ is horizontal and $V$ vertical, both of unit length, then $\left\|A_{X} V\right\|^{2}=K_{X, V}=0$. Here $K_{X, V}$ is the sectional curvature of $\operatorname{span}\{X, V\})$. This implies that $M$ is a product, cf. [O'N].

COROLLARY 4. Let $M^{n}$ be a complete noncompact manifold of nonnegative sectional curvature, $n \leq 4$. Let $S$ denote a soul of $M$.

(a) There is a Riemannian submersion $M \rightarrow S$, which splits (locally) isometrically if and only if $S$ has flat normal bundle.

(b) If the sectional curvature $K$ of $M$ is strictly positive at some point, then $M$ is diffeomorphic to Euclidean space.

Proof. (a) If $\operatorname{dim} S=3$, then the statements follow from [CG]. The case $\operatorname{dim} S=1$ is similar. In fact, (a) is true whenever $S$ is flat. We may therefore assume that $S$ is finitely covered by a 2 -sphere. If $\nu(S)$ is not flat, then (a) follows from [W, Theorem 1.2]. Otherwise, consider the universal covering $\rho: \hat{M} \rightarrow M$ with its group $\Gamma$ of covering transformations. Notice that $M \supset C$ is compact totally convex $\Leftrightarrow \rho^{-1}(C)$ is compact totally convex, and that $\rho^{-1}\left(C^{\max }\right)=\left[\rho^{-1}(C)\right]^{\max }$. It follows that $\hat{S}:=\rho^{-1}(S)$ is a soul of $M$, and $\Gamma$ preserves the splitting $\hat{S} \times P_{2}$ guaranteed by 1.3. Thus $\pi: \hat{S} \times P_{2} \rightarrow \hat{S}$ induces $M \rightarrow S$.

(b) If there is a metric splitting, there is nothing to prove. Otherwise, $\operatorname{codim} S=$ 2 , and we have a nontrivial submersion $M \rightarrow S$. But then for any $q \in M \backslash S$, $K_{u, v}=0$, where $u$ is the initial tangent vector to the minimal geodesic from $q$ to $S$, and $v \in M_{q}$ is horizontal, cf. [W]. If $q \in S$, then $K_{u, v}=0$, for any $u \in S_{q}, v \perp S_{q}$, cf. [CG]. Thus if $K$ is strictly positive at some $q$, then $S$ must be a point.

COROLLARY 5. Let $M^{n}$ be a complete noncompact manifold of nonnegative curvature, with soul $S$ of codimension 2. Then (a) and (b) of Corollary 4 remain true.

ProOF. The argument is similar to the one above. We need only consider the case when $S$ has flat normal bundle. Let $\rho: \hat{M} \rightarrow M$ denote the universal covering of $M$, with its group $\Gamma$ of covering transformations. Since the inclusion $S \rightarrow M$ is a homotopy equivalence, $\hat{S}:=\rho^{-1}(S)$ is simply connected, and by [CG, Theorem 9.1], $\hat{S}$ splits metrically as $S_{0} \times R^{k}$, with $S_{0}$ compact. By total convexity, lines in $\hat{S}$ are also lines in $\hat{M}$, and therefore $\hat{M}=M_{0} \times R^{k}$. If $\gamma \in \Gamma$, then $\gamma_{\mid \hat{S}} \in I\left(S_{0}\right) \times I\left(R^{k}\right)$ (cf. [CG]), and consequently $\gamma \in I\left(M_{0}\right) \times I\left(R^{k}\right)$. Here $I(M)$ denotes the isometry group of $M$. Now $S_{0}$ is a soul of $M_{0}$, has codimension 2 , and Theorem 3 yields a splitting $M_{0}=S_{0} \times P_{2}$. Writing $\gamma=\left(\gamma_{1}, \gamma_{2}\right) \in I\left(M_{0}\right) \times I\left(R^{k}\right)$, we see that as in Corollary $4, \gamma_{1} \in I\left(S_{0}\right) \times I\left(P_{2}\right)$. Thus the projection $S_{0} \times R^{k} \times P_{2} \rightarrow S_{0} \times R^{k}$ induces $M \rightarrow S$.

Let us finally point out that, in view of the above results, it seems reasonable to ask whether any complete, noncompact $M$ with $K \geq 0$ admits a Riemannian submersion onto its soul. 


\section{REFERENCES}

[CG] J. Cheeger and D. Gromoll, On the structure of complete manifolds of nonnegative curvature, Ann. of Math. (2) 96 (1972), 413-443.

[H] R. Hermann, On the differential geometry of foliations, Ann. of Math. (2) 72 (1960), 445-457.

[O'N] B. O'Neill, The fundamental equations of a submersion, Michigan Math. J. 13 (1966), 459469.

[W] G. Walschap, Nonnegatively curved manifolds with souls of codimension 2, J. Differential Geom. 27 (1988), 525-537.

[Y] J.-W. Yim, Distance nonincreasing retraction on a complete open manifold of nonnegative sectional curvature, preprint.

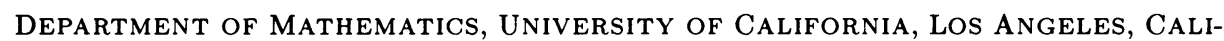
FORNIA 90024-1555 